\title{
Análisis sobre comportamientos éticos en la educación virtual
}

Eucario Parra*

Recibido: 03-08-2020

Aceptado: 03-09-2020

Citar como: Parra, E. (2021). Análisis sobre comportamientos éticos en la educación virtual. Revista Interamericana de Investigación, Educación y Pedagogía, 14(2), $113-140$. https://doi. org/10.15332/25005421.6059

\section{Resumen}

El objetivo de la investigación fue analizar las percepciones sobre comportamientos éticos en los ambientes virtuales de aprendizaje de educación superior. Se utilizó un enfoque metodológico mixto con técnicas cualitativas para comprender las impresiones de sus estudiantes, profesores y egresados sobre la autenticidad, alteridad, sentimientos y conductas en los procesos formativos; además, se usaron técnicas estadísticas para conocer las opiniones extrínsecas sobre su credibilidad y confianza. Se aplicaron dos encuestas, tres grupos focales y nueve entrevistas en profundidad. Los resultados mostraron que hacia afuera de la educación virtual hay desconfianza sobre la autenticidad y conducta de los participantes, mientras que internamente se hallaron perspectivas sobre los compromisos éticos de los participantes en procesos de aprendizaje y gestión institucional. Se encontró que, en los ambientes virtuales, los profesores, estudiantes, directivos y gestores deben demostrar autonomía responsable consigo mismos y con los otros. Con base en los hallazgos se propuso un código de ética para la educación virtual.

Palabras clave: aprendizaje en línea, enseñanza programada, ética, método de aprendizaje, responsabilidad del docente, tecnología educacional.

* Corporación Universitaria Minuto de Dios.

Correo electrónico: eucarioparra5@gmail.com

ORCID: https://orcid.org/0000-0002-5623-7440 


\title{
Analysis of Ethical Behaviors in Virtual Education
}

\begin{abstract}
The objective of the research was to analyze perceptions about ethical behaviors in virtual learning environments of higher education. A mixed methodological approach with qualitative techniques was used to understand impressions of students, teachers, and graduates on authenticity, alterity, feelings, and behaviors in the formative processes. Besides, statistical techniques were used to ascertain extrinsic opinions on their credibility and trustworthiness. Two surveys, three focus groups, and nine indepth interviews were conducted. The results showed that outside of virtual education there is mistrust about the authenticity and conduct of the participants, while, internally, perspectives were found on the ethical commitments of the participants in learning processes and institutional management. It was found that, in virtual environments, teachers, students, directors, and managers must demonstrate responsible autonomy with themselves and with others. Based on the findings, a code of ethics for virtual education was proposed.
\end{abstract}

Keywords: online learning, programmed teaching, ethics, learning method, teacher responsibility, educational technology.

\section{Análise sobre comportamentos éticos na educação virtual}

\section{Resumo}

O objetivo da pesquisa foi analisar as percepções sobre comportamentos éticos nos ambientes virtuais de aprendizagem de educação superior. Utilizou-se uma abordagem metodológica mista 
com técnicas qualitativas para compreender as impressões de estudantes, professores e egressados sobre a autenticidade, alteridade, sentimentos e condutas nos processos formativos. Além disso, foram aplicadas técnicas estatísticas para saber as opiniões extrínsecas sobre credibilidade e confianza. Foram realizadas duas enquetes, três grupos de discussão e nove entrevistas detalhadas. Os resultados mostraram que fora da educação virtual existe uma desconfiança sobre a autenticidade e a conduta dos participantes, enquanto internamente foram encontradas perspectivas sobre os compromissos éticos dos participantes em processos de aprendizagem e gestão institucional. Descobriu-se que, nos ambientes virtuais, os professores, os estudantes, os diretores e os gestores devem demonstrar autonomia responsável consigo mesmo e com os demais. Com base nas descobertas, propõe-se um código de ética para a educação virtual.

Palavras-chave: aprendizagem on-line, ensino programado, ética, método de aprendizagem, responsabilidade do docente, tecnologia educacional.

\section{Introducción}

Los ambientes virtuales de aprendizaje son escenarios para intercambiar conocimientos, distribuir información, aplicar enseñanzas, evaluar la consecución de logros y garantizar la autenticidad y transparencia de los actores (Cando, Alcoser, Villa y Ramos, 2017). En estos ambientes los estudiantes seleccionan de manera autónoma sus entornos personales de aprendizaje (PLE, por sus siglas en inglés) con recursos físicos, tecnológicos, procesos de aprendizaje y comunicacionales (Leiva, Cabero y Ugalde, 2018). De igual modo, seleccionan e integran los PLE para ampliar conocimientos y desarrollar sus actividades de aprendizaje (Chaves-Barboza y Sola-Martínez, 2018). Estos entornos tienen mayor trascendencia formativa que las plataformas o sistemas 
de gestión de aprendizaje (LMS, por sus siglas en inglés), porque están sustentados en la autodeterminación de los estudiantes, mientras los otros concentran contenidos y decisiones del profesor. Al construir los PLE, los estudiantes deciden sobre sus compromisos, los cuales, en algunos casos, están condicionados por la evaluación y, en otros, por su gusto. De manera indistinta, son necesarias actitudes reflexivas sobre la veracidad de las fuentes de información (Serrano et ál., 2019).

Otro activador es el comportamiento del profesor en el ambiente virtual. Las tecnologías de la información y la comunicación (TIC) facilitan la transformación consciente de sus papeles y competencias para orientar, comunicar y complacer al estudiante. Asimismo, deben utilizarlas de manera hábil para el aprendizaje colaborativo, pluralista, significativo y diseminado en nodos. El docente no es un técnico integrador de informática, sino que sus compromisos esenciales son organizar, dinamizar intersubjetividades y promover el trabajo colaborativo. La médula de los cursos virtuales es el aprendizaje y no la tecnología; es un acuerdo implícito y, por ende, es responsabilidad ética del profesor utilizar mediaciones para el ser estudiante. Su actitud constructiva es un cometido que involucra la pedagogía y la conducta de los profesores (Martínez et ál., 2018). El constructivismo implica que los estudiantes elaboran sus propios procesos de aprendizaje, experimentaciones y creatividades (Cañón, 2018), característica que naturaliza los ambientes virtuales.

Con cierta recurrencia en los ámbitos académicos y sociales - y debido a las intersubjetividades sobre educación tradicionalaparecen interrogantes sobre empatías entre los participantes en la virtualidad y la manera como construyen transformaciones individuales, como ocurre en la institución tradicional. Las cuestiones tocan también la ética institucional alrededor de asuntos como la deserción estudiantil, la masificación de la cobertura educativa o la autenticidad de los participantes. Se admite que el abandono es una dificultad en esta modalidad educativa (Pedró, 2020). Las autoevaluaciones deben explicar las relaciones entre dicho fenómeno 
y las debilidades en la acogida a estudiantes y la socioafectividad en el entorno virtual.

La ampliación de la cobertura educativa en la virtualidad es irregular cuando se menoscaba el criterio pedagógico por privilegiar la masificación, sembrándose así incertidumbres sobre la conducta institucional. Es el caso de los cursos masivos y abiertos, los cuales se caracterizan por su automatización, masificación, mínima retroalimentación, limitada interactividad, verificación débil de los aprendizajes, énfasis informacional y acompañamiento docente limitado (Fernández-Ferrer, 2019); y porque dan prelación a enfoques pedagógicos rígidos y cerrados (Raposo-Rivas, Sarmiento y MartínezFigueira, 2017). Con fines de inclusión, el proyecto educativo virtual debe dar mayor protagonismo al profesor, que al medio tecnológico (Morales, 2017).

En relación con la autenticidad, los interrogantes enfocan la autonomía responsable de profesores, estudiantes, directivos y gestores dentro del entramado educativo virtual. Sabiendo que la autogestión y el teletrabajo sustentan a su organización, entonces debieran garantizarse mecanismos que, remotamente, cultiven valores axiológicos. En la institución virtual, el estudiante construye su proyecto de vida cuando comprende conscientemente "sus valores, capacidades, habilidades y competencias" (Santana-Vega, Medina-Sánchez y Feliciano-García 2019, p. 424). La intencionalidad axiológica puede proveerse al incorporar institucionalmente una pedagogía de la alteridad superpuesta por tres dimensiones pedagógicas: la relación sensible, la escucha y la invitación (Vila, 2019). La alteridad es acoger a los estudiantes y promover la valoración del otro con sus singularidades, e implica comprender que los estudiantes requieren satisfactores de directivos, gestores y profesores. El ámbito virtual es un encuentro de personas y por ello el compromiso ineludible de provocar la responsabilidad de los estudiantes consigo mismos a partir del diálogo y la comunicación con los otros (Mendoza, 2019).

La ética académica en la virtualidad no se reduce al riesgo del fraude, pues la institución no está exenta del propósito de formación 
para la convivencia social y la construcción de ciudadanía (Avendaño, Paz y Parada, 2016). Los entornos virtuales deben ser escenarios para la socioafectividad, la estética, el compañerismo y la creatividad (Melo-Solarte y Díaz, 2017). Martínez (2014) encontró comportamientos deshonestos en los ambientes virtuales y la necesidad de identificar valores y principios éticos en la institución. El investigador presenta unos principios básicos: "honestidad, responsabilidad, respeto, justicia, trabajo colaborativo, disciplina, autorregulación, compromiso y solidaridad" (2014, p. 128) y exhibe estudios sobre comportamientos irregulares de los estudiantes en los ambientes virtuales. Afirma que la mente ética, la mente respetuosa, la mente disciplinada y la mente creativa son capacidades cognoscitivas para los retos del siglo XXI. La ética digital debe cuidar el buen actuar ante los intentos de plagio, fraude o suplantación de identidades, y es otra competencia transversal, como la creatividad (Martínez, 2017). Sanromán, Gonzales y Villa (2015) afirman que la ética tiene un marco general y por eso sería infructuoso codificar reglas sobre lo permitido o no en situaciones específicas, porque cada persona tiene su impronta moral. Un código de ética representa una esperanza axiológica sobre comportamientos en la virtualidad educativa (Cifuentes-Muñoz, 2019). La institución virtual debe promover el comportamiento ético en relación con la contemplación de riesgos, control de contenidos y prevención del plagio (Zapata-Ríos, 2018).

El problema de la investigación lo constituyó el comportamiento ético en los ambientes virtuales. La educación virtual promulga la libertad de los estudiantes para su desarrollo académico y personal, con autodeterminación y disciplina. Pero su conducta puede ser influenciada por la de otros actores. La investigación fue motivada por la necesidad de reconocer subjetividades en la educación virtual, para la viabilidad de un código de ética que sea fundamento para determinaciones de los participantes. El objetivo del proyecto fue analizar las percepciones sobre los comportamientos en los ambientes virtuales de aprendizaje en el contexto de la educación superior. 


\section{Método}

Se aplicó un método de enfoque mixto con técnicas cualitativas y cuantitativas. La indagación cuantitativa se utilizó con dos propósitos: apoyar la construcción de instrumentos cualitativos aplicados en las entrevistas y grupos focales, y recoger percepciones de público extrínseco sobre la confianza que genera la educación virtual. El componente cualitativo utilizó un diseño fenomenológico para reconocer experiencias y sentimientos de profesores, estudiantes y egresados de la educación virtual, así como de expertos en educación superior sobre dinámicas formativas y comportamientos en la educación virtual. Se buscó entender el significado que los actores le dan a la subjetividad y a la ética en los ambientes virtuales, a partir de sus experiencias, conocimientos y opiniones. Para la propuesta del código de ética se hizo una revisión documental sobre declaraciones de código en 30 organizaciones, con el objetivo de estudiar sus estructuras, concepciones y perspectivas diversas.

Dentro del componente cualitativo se realizaron tres grupos focales con ocho participantes cada uno, integrados por estudiantes, egresados y profesores de educación virtual de pregrado y de posgrado de nueve instituciones de educación superior. También se aplicaron nueve entrevistas en profundidad a expertos en educación superior (rectores, vicerrectores académicos, investigadores y coordinadores de educación virtual). Se tuvo en cuenta la apreciación de los egresados sobre los comportamientos en los ambientes virtuales, por sus vivencias directas y recorrido. Los testimonios de los estudiantes se consideraron significativos para conocer sus convivencias, comportamientos, compromisos y posturas con respectos a la subjetividad en esos ambientes. Por su parte, los profesores fueron invitados por ser el eje orientador del aprendizaje y por su relación directa con los estudiantes. Los expertos se tuvieron en cuenta porque su recorrido y conocimientos sobre el dinamismo de la educación superior podrían servir como fuente de validación y por sus conceptos objetivos como observadores. 
Como medio para recoger los datos cualitativos en los grupos focales y entrevistas se esgrimieron preguntas detonadoras relacionadas con: 1) la forma como se tejen la alteridad y la convivencia en el ambiente virtual, y el reconocimiento del otro aun estando distante; 2) sentimientos sobre autenticidad, validación y verificación de sujetos, y actuaciones en la virtualidad; 3 ) comportamientos de profesores y estudiantes en las actividades de aprendizaje y corresponsabilidad con los compromisos; 4) los roles de estudiantes, profesores, directivos y gestores en el ambiente virtual y la manera como son compartidos; y la 5) gratificación con los ambientes virtuales de aprendizaje y credibilidad sobre los procesos formativos que allí se desarrollan.

Para la obtención de datos cuantitativos se aplicaron dos encuestas. En la tabla 1 se muestran las especificaciones.

Tabla 1. Diseño cuantitativo.

\begin{tabular}{|c|c|c|}
\hline Instrumento & Estratificación & Variables centrales \\
\hline $\begin{array}{l}\text { Encuesta } 1 \\
\text { Muestra: } 589 \\
\text { Margen de error: } 4.04 \% \\
\text { Nivel de confianza: } 95 \% \\
\text { Número de cuestionarios: } 1\end{array}$ & $\begin{array}{l}\text { Estudiantes de pregrado de } \\
\text { universidad privada presencial } \\
\text { Estudiantes de posgrado de } \\
\text { universidad privada presencial } \\
\text { Estudiantes de pregrado de } \\
\text { universidad pública presencial } \\
\text { Estudiantes de bachillerato } \\
\text { presencial }\end{array}$ & $\begin{array}{l}\text { Imaginario en el medio } \\
\text { universitario } \\
\text { Imaginario en educación media }\end{array}$ \\
\hline $\begin{array}{l}\text { Encuesta } 2 \\
\text { Muestra: } 325 \\
\text { Margen de error: } 5.4 \% \\
\text { Nivel de confianza: } 95 \% \\
\text { Número de cuestionarios: } 2\end{array}$ & $\begin{array}{l}\text { Personas vinculadas con el } \\
\text { sector empresarial } \\
\text { Personas con capacidad de } \\
\text { decisión en hogares }\end{array}$ & $\begin{array}{l}\text { Imaginario en el medio } \\
\text { empresarial } \\
\text { Imaginario en hogares }\end{array}$ \\
\hline
\end{tabular}

Fuente: proyecto ética y calidad en la educación virtual.

Se consideraron los primeros tres estratos, porque su estadía en el campus físico y el vínculo directo con la comunidad académica posibilitan criterios basados en vivencias para formular discrepancias y comparaciones. Aunque no conviven en la educación virtual, son conocedores de los procesos y vivencias de la educación superior 
y por eso adquieren cierta autoridad en sus opiniones. El grupo de estudiantes de bachillerato fue invitado por su percepción basada en expectativas de conocer las dimensiones reales de la vida universitaria. Sus opiniones representan el nivel de confianza que les despierta la educación en ambientes virtuales. Los dos últimos estratos encarnan la opinión del ciudadano común, no relacionado directamente el sector educativo, pero que pueden ser incidentes en las decisiones de otros. En el proceso de la interpretación final se decidió realizar un análisis de tipo convergente de datos cualitativos y cuantitativos (figura 1).

Figura 1. Ruta del análisis.

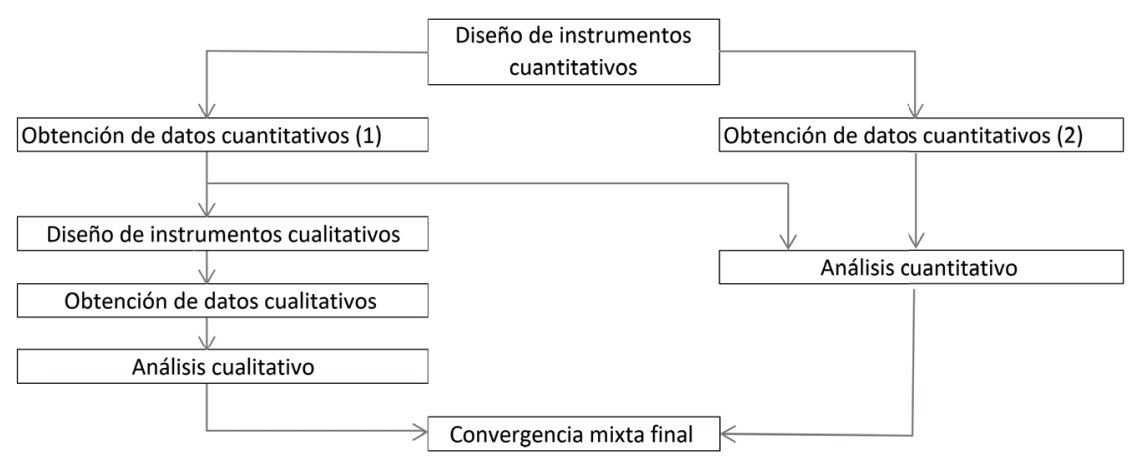

Fuente: elaboración propia.

En el análisis cualitativo se entrelazaron las siguientes categorías configuradas durante la interpretación de textos y la construcción de una red semántica: 1) compromiso y disposición de estudiantes y profesores con los procesos de aprendizaje; 2) comportamientos de gestores, directivos y profesores en la acogida a los estudiantes; y 3) autenticidad y conducta de los estudiantes y profesores. Con respecto a las estrategias para el análisis cualitativo, este se realizó con una lógica inductiva consistente en la evaluación reiterada y en espiral de los textos arrojados por las entrevistas y grupos focales. Se fueron seleccionando así nodos y relaciones de forma de red semántica para ir constituyendo las unidades de análisis o categorías. El análisis cuantitativo de los datos se hizo con base en la siguiente hipótesis descriptiva: la educación virtual genera desconfianza 
en el medio social sobre la autenticidad de los comportamientos de estudiantes y profesores.

Este artículo corresponde a los objetivos específicos relacionados con el código de ética del proyecto Ética y calidad de la educación virtual. Los objetivos sobre calidad no se incluyeron.

\section{Resultados}

\section{Hallazgos cuantitativos}

En las tablas 2 y 3 se muestran los hallazgos sobre el comportamiento ético de profesores y estudiantes, diferenciados por los siguientes estratos: estudiantes de pregrado de universidad privada presencial (A); estudiantes de posgrado de universidad privada presencial (B); estudiantes de pregrado de universidad pública presencial (C), estudiantes de bachillerato presencial (D).

Tabla 2. Percepciones sobre comportamiento de los estudiantes.

\begin{tabular}{|l|l|l|l|l|}
\hline \multirow{2}{*}{} & \multicolumn{4}{|c|}{ Estrato } \\
\cline { 2 - 5 } & A & B & C & D \\
\hline $\begin{array}{l}\text { Los estudiantes pueden suplantar su identidad } \\
\text { fácilmente. }\end{array}$ & $61.7 \%$ & $60.9 \%$ & $60 \%$ & $60.2 \%$ \\
\hline $\begin{array}{l}\text { Los estudiantes pueden hacer fraude en las } \\
\text { evaluaciones fácilmente. }\end{array}$ & $63.2 \%$ & $63.8 \%$ & $77.5 \%$ & $71.6 \%$ \\
\hline $\begin{array}{l}\text { Los estudiantes tienen mayor oportunidad para hacer } \\
\text { plagios. }\end{array}$ & $56.4 \%$ & $58.6 \%$ & $52.5 \%$ & $71.6 \%$ \\
\hline
\end{tabular}

Fuente: proyecto ética y calidad en la educación virtual.

Tabla 3. Percepciones sobre comportamiento de los profesores.

\begin{tabular}{|l|l|l|l|l|}
\hline \multirow{2}{*}{} & \multicolumn{4}{|c|}{ Estrato } \\
\cline { 2 - 5 } & A & B & C & D \\
\hline $\begin{array}{l}\text { Los profesores pueden mentir fácilmente a sus } \\
\text { estudiantes. }\end{array}$ & $27.1 \%$ & $26.1 \%$ & $12.5 \%$ & $45.5 \%$ \\
\hline $\begin{array}{l}\text { Los profesores pueden ocultar sus carencias } \\
\text { fácilmente. }\end{array}$ & $55.6 \%$ & $57.8 \%$ & $57.5 \%$ & $70.5 \%$ \\
\hline
\end{tabular}

Fuente: proyecto ética y calidad en la educación virtual. 
La tabla 4 exhibe los resultados sobre confianza en la educación virtual, diferenciada por los siguientes estratos: personas vinculadas con el sector empresarial (E) y personas con capacidad de decisión y responsabilidad en los hogares (F).

Tabla 4. Percepciones sobre confianza en la educación virtual.

\begin{tabular}{|l|l|l|}
\hline \multirow{2}{*}{} & \multicolumn{2}{|c|}{ Estrato } \\
\cline { 2 - 3 } & E & F \\
\hline $\begin{array}{l}\text { La educación virtual facilita el fraude y la } \\
\text { suplantación. }\end{array}$ & $57.3 \%$ & $59.4 \%$ \\
\hline $\begin{array}{l}\text { La educación virtual no genera confianza en la } \\
\text { formación profesional. }\end{array}$ & $61.5 \%$ & $49.6 \%$ \\
\hline
\end{tabular}

Fuente: proyecto ética y calidad en la educación virtual.

Las fuentes del entorno fueron estudiantes de universidad y bachillerato presencial y personas de empresas y de hogar. Con porcentajes entre $56.4 \%$ y $77.5 \%$, los universitarios creen que la educación virtual facilita suplantaciones, hay riesgo del fraude y posibilita el plagio. La opinión de los estudiantes de bachillerato sobre esos aspectos fue de $60.2 \%, 61.6 \%$ y $61.7 \%$. Los primeros conocen el ambiente universitario y por eso sus opiniones están validadas por las experiencias, mientras que los segundos son ajenos absolutamente. La hipótesis contrastada con la tabla 4 explica que hay recelos en las personas de hogar y de empresas sobre los comportamientos y la formación en la educación virtual, con resultados entre $49.6 \%$ y $61.5 \%$. Los cuatro grupos opinan sustentados en imaginarios, pues no tienen vivencias directas dentro de esa modalidad.

La contrastación con la tabla 3 en relación con los comportamientos de los profesores rechaza parcialmente la hipótesis. Los grupos de estudiantes universitarios, con datos entre $12.5 \%$ y $27.1 \%$, dijeron que en la virtualidad los profesores pueden mentir; estadísticamente es minoría y se deduce credibilidad sobre su honradez. Sin embargo, entre el $52.5 \%$ y el $58.6 \%$, afirmaron que se pueden ocultar carencias personales y profesionales en la virtualidad. Los estudiantes de bachillerato expresaron el mismo con porcentajes de $45.5 \%$ y $70.5 \%$. 


\section{Hallazgos cualitativos}

\section{Primera categoría: compromiso y disposición de estudiantes y profesores}

Los profesores mencionaros que el aprendizaje en el ambiente virtual es dinámico, pues los estudiantes proactivamente crean sus ambientes personales de aprendizaje con recursos y estrategias propias. Un profesor afirmó que "cada estudiante inventa sus ambientes de estudio y colecciona sus propios medios". Según los profesores, el aprendizaje contextualizado basado en problemas reales, con significado para el estudiante, incrementa su responsabilidad. Un tema redundante en los grupos focales fue el trabajo colaborativo. Unos egresados lo destacaron, mientras otros lo criticaron mencionando que las instituciones lo imponen; pero la participación diáfana queda viciada por coyunturas individuales. Según los profesores, los equipos de trabajo en la modalidad virtual son creativos, investigativos, interculturales y sirve como mecanismo de autorregulación entre los compañeros.

Otras divergencias se notaron en la concepción sobre foros virtuales y rúbricas. Los estudiantes afirmaron que participan porque es requisito de evaluación, pero las motivaciones iniciales se desvanecen por la pasividad de los profesores. Para ellos, en cambio, los foros son la expresión comunicativa máxima del ambiente virtual. Las rúbricas, según unos profesores, representan la evaluación formativa y la apreciación pedagógica. Otros criticaron su implementación, porque hay instrucciones que las imponen con formatos rígidos y extensos. Un estudiante aseveró: "las rúbricas las utilizan algunos profesores, pero se ve que improvisan, pues hay veces que los formularios no tienen que ver nada con la actividad entregada".

De acuerdo con los profesores, el dinamismo de los estudiantes frente a las actividades académicas es señal de rectitud, honestidad y comportamiento coherente; de manera contraria, sería transgredir su proyecto de vida. Egresados, estudiantes, profesores y expertos coincidieron en que el estudiante virtual le da prelación 
a sus compromisos laborales o familiares, quedando el cumplimiento académico expuesto a coyunturas. Sin embargo, destacaron su espíritu exploratorio y su capacidad para organizar información. Según un vicerrector: "las instituciones virtuales tienen que entender que su público son trabajadores que estudian y no estudiantes que trabajan". Consideró faltos de ética a profesores y directivos cuando ignoran las particularidades de los estudiantes.

Por su parte, los profesores afirmaron que es fundamental la mediación pedagógica de la tecnología y que las plataformas sean centro de aprendizaje y comunicación. Enfatizaron en la lealtad del profesor, su ética y dedicación para los procesos educativos virtuales. Los egresados fueron amplios y generosos con los profesores; no obstante, uno afirmó: "tuve profesores con pereza pedagógica, hacían lo simple y cumplían con lo más básico, pero no aportaban más. En la virtualidad los profesores son una esperanza para los estudiantes y por eso defraudan cuando no dan mucho". Para los estudiantes, sus profesores no son la fuente exclusiva de información, pero sí la más influyente.

\section{Segunda categoría: comportamientos de gestores, directivos y profesores}

En las entrevistas, los expertos afirmaron que el reto para los directivos es lograr la comprensión social de la tecnología para la educación. Es decir, que se gane confianza en la gente desde la calidad de los cursos virtuales y que se propicie un cambio cultural y socioeconómico con la tecnología educativa. También destacaron que la educación virtual debe representar socialmente escenarios pedagógicos y no únicamente sistemas tecnológicos. Un rector aseveró que "no es ético traicionar la confianza de la gente en la educación virtual, ni ocultar cosas en procesos de mercadeo. Este tipo de educación no es para cualquier persona y hay que ser claros con esto".

De igual manera, varios profesores expusieron que la enseñanza requiere plataformas para aprendizaje móvil, integración 
con las redes sociales e innovaciones de inteligencia artificial. Sin embargo, indicaron que estos avances son posibles si los directivos son solidarios con los estudiantes y los profesores facilitándoles cumplir con sus compromisos. Al respecto, un profesor afirmó que "las universidades virtuales creen que la única tecnología posible son videoconferencias y Moodle, o videos que a veces nosotros mismos hacemos".

Los estudiantes y egresados subrayaron las ventajas de estudiar virtualmente: sin limitaciones de horarios y desplazamientos. Para profesores y expertos, las ventajas son la versatilidad pedagógica y el ambiente educativo transformado. Enfatizaron en la necesidad del acompañamiento individualizado a los estudiantes, porque están físicamente aislados en sus hogares.

Algunos estudiantes afirmaron sentir una recepción fría y, con frecuencia, limitada de compañeros, profesores y directivos; aunque dos señalaron casos de amistades surgidas entre estudiantes y fraternidad con algunos profesores. Varios egresados sintieron acogida por parte del personal de mercadeo y de comunicaciones, pero indiferencia por parte de los demás directivos, así como acogida parcial de sus profesores. Afirmaron que gestores y directivos mostraban acogida solo en procesos de matrículas, cierres de periodo o atención de quejas o reclamos.

Mi esposa no aguantó, había iniciado en una universidad presencial y por motivos laborales decidió estudiar psicología virtual. Nunca se enganchó con los compañeros y vivía comparando con la otra universidad; y dice que casi todo eran documentos. La decepcionó no encontrar la tecnología ni los medios que estaba esperando para suplir las clases físicas. Es feo decirlo, pero se sintió engañada ¿Por qué? Porque la vendedora le prometió que todo era mejor, que se trabajaba en equipos y que se investigaba. Yo digo que los profesores y los decanos deben presionar para que la gente se sienta bien y no salgan hablando mal de la universidad. (Comunicación personal de un egresado) 


\section{Tercera categoría: autenticidad y conducta de estudiantes y profesores}

Según varios estudiantes, en ocasiones se presentaron fraudes en los ambientes virtuales, y relataron situaciones específicas sobre negociación de tareas, suplantaciones, simulaciones o fingimientos en las actividades de evaluación. Dijeron no sentir controles institucionales, aunque reconocieron que los profesores implementaron acciones como exposiciones en foros o en videoconferencias para asegurar la autenticidad.

No he sentido controles sobre autenticaciones. Tenemos un usuario y contraseña, pero no más. Decir que en las evaluaciones o en otras cosas hay preocupaciones por las identidades sería mentir y nosotros sabemos de casos donde hubo suplantaciones, pero no siempre es así en todas las asignaturas. (Un estudiante)

Algunos egresados afirmaron que la deshonestidad es una deficiencia también de las universidades presenciales y que la falta de ética proviene de la actitud del estudiante y no de la institución virtual. Poca importancia dieron al tema de la autenticidad e hicieron énfasis en las oportunidades de la virtualidad para la formación profesional. Estudiantes y egresados coincidieron en que la ética tiene condición personal y cada cual asume las responsabilidades académicas bajo su criterio. Afirmaron que no existe un mecanismo que garantice en un $100 \%$ evitar actos deshonestos en la virtualidad, aunque se puede mermar el riesgo si se concientiza a los estudiantes sobre la responsabilidad con su futuro. Un experto entrevistado explicó que existen medios tecnológicos que remotamente pueden detectar intentos de fraude en los exámenes o suplantaciones, pero son poco usados. Los egresados afirmaron que los estudiantes deben ser conscientes que la educación virtual es un medio para obtener conocimientos, pero no para obtener títulos fácilmente.

Según los expertos, la desconfianza observada a veces sobre la educación virtual se debe a que las personas tienen un paradigma 
de clase representado por aprendices reunidos en intramuros, controlados directamente por el profesor. Uno de los entrevistados afirmó: "antes que intentar controvertir esas opiniones, hay que plantear debates, análisis, recapitulaciones positivas". Los expertos manifestaron que la autenticidad es un asunto de la persona, no de la virtualidad. La ética es intrínseca, viene con la formación del individuo, pero es responsabilidad de las instituciones virtuales comprometerse con la integralidad del estudiante.

La ocurrencia de comportamientos dolosos es una cuestión de ética compartida y de responsabilidad de diversos actores, pues no actúa bien el estudiante que procede fraudulentamente, ni el profesor que lo facilita, ni los directivos cuando los ignoran. Un experto indicó que "es casi imposible por parte de la institución descubrir qué estudiantes o profesores están siendo suplantados".

Varios expertos afirmaron que los fraudes obedecen a procedimientos y actitudes de los profesores. Uno de los entrevistados anotó que "la validación consiste en cuánto conocimiento construye el estudiante y cómo relata esa construcción". Los estudiantes aprenden los modales del profesor, como cumplimiento, rectitud y esfuerzo; y sus conductas pueden ser influenciadas por la didáctica y la evaluación. Para varios profesores el riesgo de actuaciones deshonestas puede reducirse con el tipo de actividades: "con la aplicación social del conocimiento, en vez de repeticiones enciclopédicas, se minimiza la posibilidad de copias o trampas". El estudiante se ilusiona viéndose resolver problemas y no tiene motivos para plagios. Los profesores hicieron énfasis en que la formación basada en competencias y el aprendizaje basado en problemas transforma la conducta de los estudiantes. Un profesor, al hablar de autenticidad y honestidad, señaló que el currículo debe ahondar en la formación humanística e investigativa, porque "se van esculpiendo en los estudiantes mentalidades para ver el mundo desde su complejidad y al disfrute del conocimiento, lo que determina el regocijo frente a las actividades académicas". En general, los profesores no expresaron alarmas por riesgos de fraude o por actos deshonestos en los ambientes virtuales. 
Varios profesores destacaron que el requisito de comportamiento ético no es solo para los estudiantes, pues directivos y gestores no son éticos cuando están pensando solo en los beneficios mercantilistas o cuando ignoran la soledad de los estudiantes.

No es justo dudar solo de los estudiantes. Me perdonan por lo que digo, pero hay colegas que se programan para dar lo mínimo, para no complicarse. No dedican mucho tiempo y como son permisivos con los estudiantes y donativos, pasan desapercibidos. Mejor dicho, abusan de la virtualidad. Lo curioso es que el decano nada sabe, ni el coordinador, entonces ellos también fallan. (Un profesor)

En las entrevistas se afirmó que es cuestionable la ética de directivos y profesores en los ambientes virtuales cuando detectan problemas de aprendizaje y, aun así, los reproducen. Una falta a la ética ocurre también, según ellos, cuando el profesor desarrolla contenidos obsoletos, sin ninguna criticidad. Así lo menciona uno de los rectores:

Los directivos universitarios tenemos este gran reto de transformar vidas, de entender que esos muchachos no van a ir por unas clasecitas donde van a escuchar un discurso; esos muchachos van porque quieren aprender, porque necesitan aprender para la vida, y enfrentar unos retos que se le vienen de forma compleja. Si nosotros no estamos trabajando en esos contextos, los vamos a dejar con una educación de muy poca calidad, porque no estamos desarrollando sus avales. Ese es el reto tanto en las dos modalidades, presencial y virtual.

Sobre la viabilidad de un código de ética, los profesores no preponderaron ni desestimaron ese código, pues no reflejaría armonía y educabilidad del ambiente virtual. Un profesor afirmó: "con o sin código, el compromiso de la universidad virtual es formar personas de bien, que se respeten ellos mismos y a lo establecido". Al respecto, otro de los profesores estacó: "no es necesario desgastarse con reglamentos o códigos de actuaciones, la idea no es si el código 
sirve o para qué; debe existir es honestidad y convivencia entre todos y para todos".

Por su parte, los egresados desestimaron el código, porque se estaría implementado un reglamento más.

Aquí, en la educación virtual, además de aplicar toda la tecnología disponible que se tiene para sustentar trabajos, como software de vigilancia, programas detectores de copias, plataformas que permiten presentar exámenes en línea con grabación al momento de realizarlo, se debe trabajar mucho en la motivación del estudiante para su aprendizaje. (Un egresado)

En las entrevistas, varios expertos afirmaron que no es necesario codificar las conductas, ya que los casos son distintos y que la ética es una actitud individualizada. Además, se tendría que implementar un código diferente para cada nivel educativo.

Más que un código de ética para la educación virtual, se requieren prácticas éticas de los actores. Entonces uno nota que en las instituciones y en cualquier organización hay, digamos, reglamentos, protocolos, códigos, pero si eso no verdaderamente se interioriza como una práctica humana, como una práctica social, sigue siendo letra muerta. Por lo tanto, el tenerlo o no tenerlo, pues, puede ser un requisito para procesos de calidad, pero es un asunto más de forma. (Un experto en educación superior)

En las entrevistas y grupos focales se consideró inoportuno un código de ética por las siguientes razones: 1) se estaría reglamentando la ética y trayendo a la educación modelos empresariales e industriales; 2) se enfocaría la ética en recetas sobre conductas buenas y malas; 3) no se tendría en cuenta que el comportamiento es una decisión individual; y 4) en la educación superior existen acuerdos tácitos y explícitos sobre buenos comportamientos. 
Para la propuesta de código de ética se hizo una revisión de 30 códigos de empresas e instituciones educativas. Se encontraron tres tendencias: 1) códigos estructurados como conjuntos de reglas precisas sobre comportamientos prohibidos; 2) códigos que tienen una dirección conceptual y reflexiva sobre el actuar de las personas; y 3) códigos que tienen una orientación pedagógica sobre el comportamiento. En cuanto a su composición, coinciden en principios éticos y reglas de comportamiento. Algunos agregan concepciones sobre ética, comportamientos esperados y dispositivos didácticos.

\section{Discusión}

Los hallazgos cuantitativos y cualitativos sobre el comportamiento ético en la educación virtual descubrieron la necesidad de iniciativas pensadas en la subjetividad. La hipótesis sobre la desconfianza en el medio social con respecto a los comportamientos en el ambiente virtual fue confirmada por las estadísticas. Asimismo, los datos cualitativos sobre sus dinámicas internas configuraron complejamente una red de intereses, expectativas y vivencias.

Autonomía e incumbencia son requerimientos personales para estar en la educación virtual. Pero es una estadía ética, por cuanto es libre, deseada, satisfactoria y cuidadosa de sí mismo y de los otros (Savater, 2010), y por la ontología de los valores: de culpa, responsabilidad, libertad, objetividad y conocimiento del yo (Crespo, 2019). Por pertenecer al sistema virtual, los estudiantes, profesores, directivos y gestores tienen corresponsabilidad con el entorno y con los movimientos curriculares internos.

No sería ético que la institución permanezca impasible ante percepciones externas, pues el contexto es insumo y propósito de sus proyecciones. Los datos cuantitativos son desfavorables para la educación virtual; el escepticismo puede ser porque desconocen su evolución pedagógica, la reducen a educación automática por internet, conocen filtraciones de conductas deshonestas o ignoran 
los avances como analíticas de aprendizaje basadas en datos o aprendizajes ubicuos con tecnología móvil (Gros, 2018). En el entorno difícilmente se comprende cómo la implementación de la inteligencia artificial con analística de datos puede emparejar procesos educativos de las aulas físicas (Ruipérez-Valiente, 2020).

Con base en los anterior, el análisis cualitativo configuró tres categorías:

1. Compromiso y disposición de estudiantes y profesores con los procesos de aprendizaje. De acuerdo con los testimonios, en los ambientes virtuales hay dinamismos y actitud constructiva de profesores y estudiantes. Esto lo ratifica elementos como el trabajo en equipo, el aprendizaje basado en problemas y la participación en foros o rúbricas de evaluación. Aunque se escucharon discrepancias y críticas, tal dinamismo determina el comportamiento ético de los estudiantes y profesores, porque pertenecer determina compromisos intrínsecos con el establecimiento. Es decir, participar constructivamente adquiere una dimensión axiológica, y deja de ser procedimental o instrumental.

En ciertos pasajes se dejó entrever cómo a veces profesores e institución no comprenden que ciertos estudiantes priorizan compromisos laborales o familiares, en deterioro de los académicos, y por eso buscaron la virtualidad. La incomprensión de esos casos representa una conducta censurable, ya que dichas personas con su condición fueron matriculados. Es cuando se hace indispensable la pedagogía de la alteridad como decisión institucional. Es claro que un principio en la virtualidad es la autonomía responsable de los estudiantes, pero, como en el caso de la lectura comprensiva, el contrato compromete también a los profesores y a la institución (Tovar Vergara, 2019). 
Sin embargo, este propósito, más que un tecnicismo didáctico, es un comportamiento ético esperado.

2. Comportamientos de gestores, directivos y profesores. Su razón de ser son los estudiantes y, entonces, se transgrede la ética institucional cuando la acogida sobresale solo en procesos de mercadeo o matrículas. Es un asunto ético corresponder a la confianza de los estudiantes y satisfacer cuidosamente las necesidades de formación, socialización y comunicación. El desafío institucional - lo afirmó un rector- es evitar el reduccionismo al sistema tecnológico, entendiendo el ambiente virtual como un escenario pedagógico y social. Por ejemplo, desarrollos tecnológicos, como los sistemas basados en procesamiento de datos masivos para apoyar la enseñanza-aprendizaje, implican estrategias para el tratamiento ético de la información, para que los beneficios no vayan en detrimento de la privacidad de los estudiantes (Aznarte, 2020).

3. La autenticidad y la conducta en el ambiente virtual tejieron complejidades sobre comportamientos y expectativas. Varios estudiantes relataron vivencias sobre actos deshonestos y dijeron no sentir controles institucionales. Profesores y egresados no les dieron mucha relevancia a estos episodios. Los profesores reconocieron el riesgo por fraudes y suplantaciones y expusieron mitigaciones pedagógicas, pero fue notable su despreocupación por las implicaciones éticas. Las conductas de indiferencia, permisibilidad o anacronismo ante tales hechos son reprobables, aunque son fenomenológicos y no generalizables. La apatía institucional es de las personas, y por eso el juicio ético cae en directivos, profesores y gestores.

Así las cosas, expertos y egresados expusieron dos asuntos: la ética es de la individualidad y las actividades fraudulentas no son exclusivas de la educación virtual. Sin embargo, estas realidades 
no justifican la indeterminación institucional, porque se inhabilitarían esperanzas de formación integral y efectos trasformadores de los estudiantes. Sería inverisímil ignorar el riesgo tecnológico, porque la fácil manipulación, disponibilidad, accesibilidad de artefactos y sistemas pueden motivar prácticas deshonestas. Quienes participan en el ambiente virtual deben ser armoniosos con la ética digital, pues los sujetos producen y consumen información que, aunque moralmente neutra, puede tomar la dirección que determine el individuo.

Profesores y directivos deben asumir que un propósito básico es formar para la convivencia social. Las actitudes pasivas o elusivas de directivos, gestores y profesores de la educación virtual demandan reproches y reflexiones si desconocen al ser estudiante, puesto que él representa un compromiso ético. Estos propósitos contravienen al profesor tecnófilo, pues se esperan unos discursos diversos, multiculturales, contextualizados (Dorfsman, 2018). El profesor virtual debe asumir éticamente la integración y construcción de procesos de aprendizaje (Martínez, Pérez y Martínez, 2015). Tales propósitos involucran además a directivos y gestores, pues sistémicamente sus conductas inciden en los profesores.

\section{Código de ética}

La convergencia de los resultados cuantitativos y cualitativos justificó una propuesta de código de ética para la educación virtual. Los participantes de grupos focales y entrevistas no aprobaron la idea del código de ética, pues lo relacionaron con reglamentación de conductas y normativas de estilo empresarial. No comprendieron que puede ser un instrumento de orientación pedagógica o un marco conceptual para sustentar comportamientos — que es teleológicamente distante de enfoques punibles-, ni identificaron al código como una esperanza axiológica para el comportamiento cuidadoso y armónico. La ética significa libertad, responsabilidad, corresponsabilidad (Betancur, 2016), así que la mente ética en la virtualidad opta entre infringir la armonía del sistema o conseguir el logro sin escrúpulos. 
Se propuso el código porque los resultados estadísticos del estudio develaron la necesidad de estrategias para incrementar la confianza social en la educación virtual. A su vez, los hallazgos cualitativos mostraron la necesidad de mecanismos de mitigación, seguimiento, retroalimentación, reflexión acerca de la conducta de los actores., pues son responsables éticamente de sus actos, omisiones y desafíos (Polo, 2019). El código debe ser compartido, connatural para todos, construido colectivamente en las organizaciones (Arredondo, De La Garza y Villa, 2014).

El propósito del código de ética fue definir pautas para el comportamiento armónico y virtuoso de gestores, directivos, estudiantes y profesores en el ambiente virtual. Su estructura comprende: 1) desarrollo del comité institucional de ética; 2) perspectivas éticas de la institución, el profesor y el estudiante; 3) principios éticos: proyecto de vida, responsabilidad, libertad, corresponsabilidad, autenticidad, validación y verificación de las conductas en el ambiente virtual; 4) enfoque compartido de autodeterminaciones de estudiantes, profesores, directivos y gestores; y 5) metodología, que comprende la definición de actividades para la comunicación con los participantes, autoevaluación integral, pedagogía y continuidad del enfoque compartido.

\section{Conclusiones}

El público extrínseco a la educación virtual expresó dudas sobre la calidad y el comportamiento ético de sus actores. Estas percepciones deben ser interpretadas por planeadores y ejecutores, pues las organizaciones inteligentes leen percepciones internas y externas para su desarrollo. Por su parte, profesores, estudiantes y egresados configuraron un relacionamiento complejo de realidades y expectativas sobre la autonomía responsable y la ética en el ambiente virtual. Los expertos en educación superior manifestaron confianza en la virtualidad, expusieron correctivos y subrayaron que la subjetividad y la pedagogía son factores esenciales, sin demeritar al sistema tecnológico. 
La educación virtual avanza tecnológicamente con adelantos como la analítica de datos y el aprendizaje móvil. Junto con esto, las instituciones deben reconocer que son concentraciones de personas expectantes y ávidas de reconocimiento, que han decidido pertenecer a un ambiente virtual para satisfacer sus necesidades formativas. Aunque, al parecer es un tema manido, en la representación social de la educación virtual prevalece el sistema tecnológico sobre el educativo y por eso las suspicacias observadas.

Un código de ética para la educación virtual tiene misión distinta a la de ser una normativa para juzgar comportamientos buenos o malos. Tiene dimensiones pedagógicas y axiológicas para la reflexión de los participantes sobre sus decisiones y consecuencias. Las instituciones deben configurar sus códigos de ética para que directivos, gestores, profesores y estudiantes tengan un referente institucional para el cuidado de sus comportamientos.

\section{Referencias}

Arredondo, F., De la Garza, J. y Villa, L. (2015). Propuesta para el diseño de un código de ética empresarial basado en la ética kantiana [Proposal to design a business code of ethics based on kantian ethics]. Cuadernos de Administración, 30(52), 9-19. https://www.redalyc.org/articulo. oa? $\mathrm{id}=225033236002$.

Avendaño, W., Paz, L., Parada, A. (2016). Construcción de ciudadanía: un modelo para su desarrollo en la escuela. [The construction of citizenship: a model for its development in the school]. AGO USB, 16(2), 479-492. http://revistas.usbbog.edu.co/index.php/Agora/article/ download/2444/2319

Aznarte, J. L. (2020). Consideraciones éticas en torno al uso de tecnologías basadas en datos masivos en la UNED [Ethical considerations on the use of massive databased technologies in UNED]. RIED. Revista Iberoamericana de Educación a Distancia, 23(2), 237-252. http://dx.doi. org/10.5944/ried.23.2.26590 
Betancur, G. (2016). La ética y la moral: paradojas del ser humano. [Ethics and moral: Human being paradoxes]. Revista CES Psicología, 9(1), 109-121. http://www.redalyc.org/articulo.oa?id=423545768008

Cando, A., Alcoser, F., Villa, H. y Ramos, R. (2017). Los entornos virtuales un plus en la docencia universitaria de la Escuela Superior Politécnica de Chimborazo [The virtual environments. A plus in the university teaching of the Escuela Superior Politécnica de Chimborazo]. 3C TIC, 6(3), 26-42. http://dx.doi.org/10.17993/3ctic.2017.57.26-42.

Cañón, A. L. (2018). Desplazamiento de los docentes de lengua castellana a otras prácticas profesionales. Revista Interamericana de Investigación, Educación y Pedagogía (RIIEP), 13(1), 207-235. https:// doi.org/10.15332/s1657-107X.2018.0002.06

Crespo, M. (2019). La contribución de Rudolf Otto a la ética. Ideas y Valores, 68(170), 75-97. 10.15446/ideasyvalores.v68n170.62460

Cifuentes-Muñoz, A. (2019). Epistemología implícita en el código de ética profesional del colegio de psicólogos de Chile [Implicit epistemology in charter of professional ethics for psychologists in Chile]. Cinta de Moebio, 64, 51-67. http://dx.doi.org/10.4067/s0717-554x2019000100051

Chaves-Barboza, E. y Sola-Martínez, T. (2018). Entornos personales de aprendizaje (PLE) en el Grado de Educación Primaria de la Universidad de Granada [Personal Learning Environments (PLE) in the Bachelor's Degree in Elementary Education at the University of Granada]. Revista Electrónica Educare, 22(1), 1-18. http://dx.doi.org/10.15359/ree.221.12

Dorfsman, M. (2018). El educador en línea: más allá de la digitalidad [The online educator: beyond digitaly]. RED, Revista de Educación a Distancia, 56(9), 1-25. http://dx.doi.org/10.6018/red/56/9

Fernández-Ferrer, M. (2019). Revisión crítica de los Mooc: pistas para su futuro en el marco de la educación en línea [A critical review of Mooc: clues for their future in the framework of online learning] Revista de Docencia Universitaria, 17(1), 73-88.:https://doi.org/10.4995/redu.20 19.11275 
Gros, B. (2018). La evolución del e-learning: del aula virtual a la red [The evolution of e-learning: from virtual classroom to the network]. RIED, Revista Iberoamericana de Educación a Distancia, 21(2), 69-82. http:// dx.doi.org/10.5944/ried.21.2.20577

Leiva, J, Cabero, J. y Ugalde, L. (2018). Entornos personales de aprendizaje (PLE) en estudiantes universitarios de Pedagogía. [Personal Learning Environments (PLE) in College Students of Pedagogy]. Revista Latinoamericana de Tecnología Educativa, 17(1), 25-39. https://doi. org/10.17398/1695-288X.17.1.25

Martínez, C., Pérez C. y Martínez J. (2015). Las TIC y el entorno virtual para la tutoría universitaria [Icts and virtual environments for university tutoring]. Educación XX1, 19(1), 287-310. https://doi.org/10.5944/ educxx1.13942

Martínez, E. (2014). Hacia un código de ética para la educación virtual, basado en las cinco mentes del futuro. [Towards an Ethics Code for Virtual Education, Based on The Five Minds for the Future]. Revista mexicana de bachillerato a distancia, 6(11), 127-134. http://dx.doi.org/10.22201/ cuaed.20074751e.2014.11.65017

Martínez, O., Steffens, E. J., Ojeda, D. C. y Hernández, H. G. (2018). Estrategias pedagógicas aplicadas a la educación con mediación virtual para la generación del conocimiento global [Pedagogical strategies applied to education with virtual media for the generation of global knowledge]. Formación universitaria, 11(5), 11-18. http://dx.doi. org/10.4067/S0718-50062018000500011

Martínez, X. (2017). Onlife manifesto y la ética digital [Onlife Manifesto and digital ethics]. Revista Mexicana de Bachillerato a Distancia, 9(18), 15-22. http://dx.doi.org/10.22201/cuaed.20074751e.2017.18.64926.

Melo-Solarte, D. y Díaz, P. (2018). El Aprendizaje Afectivo y la Gamificación en Escenarios de Educación Virtual [Emotional learning and gamification in virtual education environments]. Información tecnológica, 29(3), 237248. https://dx.doi.org/10.4067/S0718-07642018000300237. 
Mendoza Rivera, W. F. (2019). La Pedagogía de la Respuesta como modelo pedagógico. La formación humanista Tomasiana-Sedaniana. Revista Interamericana de Investigación, Educación y Pedagogía (RIIEP), 13(1), 207-235. https://doi.org/10.15332/25005421/5467

Morales, N. (2017). Las TIC y los escolares del medio rural. Entre la brecha digital y la educación inclusiva [ICT and pupils of rural areas: Between the digital gap and inclusive education]. Revista de Pedagogía, 69(3), 41-56. http://orcid.org/0000-0002-0048-2607

Pedró, F. (2020). Perspectiva internacional del aseguramiento de la calidad [ponencia]. II Foro Internacional Sobre el Aseguramiento de la Calidad de la Educación Superior, Bogotá, Colombia. http://unired.edu.co/ index.php/calendario/896-ii-foro-internacional-sobre-el-aseguramiento-de-la-calidad-de-la-educacion-superior

Polo, M. Á. (2019). La responsabilidad ética. [The ethical responsibility]. Veritas, (42), 49-72. https://dx.doi.org/10.4067/S0718-92732019000 100049

Raposo-Rivas, M., Sarmiento, J. y Martínez-Figueira, M. (2017). El perfil pedagógico de los Mooc a partir de un estudio exploratorio. [The pedagogic profile of the Mooc from an exploratory study]. Estudios pedagógicos, 43(2), 277-292. https://dx.doi.org/10.4067/S0718-0705 2017000200015.

Ruipérez-Valiente, J. A. (2020). El Proceso de Implementación de Analíticas de Aprendizaje [The Implementation Process of Learning Analytics]. RIED, Revista Iberoamericana de Educación a Distancia, 23(2), 85-101. http://dx.doi.org/10.5944/ried.23.2.26283.

Sanromán, R., González, I. y Villa, M. (2015). Los principios éticos y las obligaciones civiles [Ethical principles and civil obligation]. Boletín mexicano de derecho comparado, 48(142), 313-337. https://doi. org/10.22201/iij.24484873e.2015.142.4922 
Santana-Vega, L. E., Medina-Sánchez, P. C. y Feliciano-García, L. (2019). Proyecto de vida y toma de decisiones del alumnado de Formación Profesional. [Life design and decision making of vocational training students]. Revista Complutense de Educacion, 30(2), 423-440.

Savater, F. (2010). Ética para amador. Ariel.

Serrano, J. L., Carrera, X., Brescó, E. y Suárez-Guerrero, C. (2019). Tratamiento crítico de la información de estudiantes universitarios desde los entornos personales de aprendizaje [Critical processing of information on university students from personal learning environments]. Educação e Pesquisa, 45. https://dx.doi.org/10.1590/s1678-4634201945193355

Tovar Vergara, E. L. (2019). Implementación de estrategias pedagógicas constructivistas mediadas por las herramientas Web 2.0 para el fortalecimiento de la comprensión teórica en los contenidos conceptuales de las ciencias naturales y la educación ambiental. Revista Interamericana de Investigación, Educación y Pedagogía, RIIEP, 12(2), 71-112. https:// doi.org/10.15332/25005421.5009

Vila, E. (2019). Repensar la relación educativa desde la pedagogía de la alteridad [Rethinking the educational relationship from the pedagogy of alterity]. Teoría de la Educación, 31(2), 177-196. http://dx.doi. org/10.14201/teri.20271

Zapata-Ros, M. (2018). Gestión del aprendizaje y web social en la Educación Superior en línea [Learning management and social web in Higher Education online]. RED, Revista de Educación a Distancia, 57(7), 1-34. http://dx.doi.org/10.6018/red/57/7

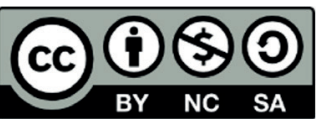

